\title{
Wear studies of hydroxyapatite composite coating reinforced by carbon nanotubes
}

\author{
Y. Chen ${ }^{\mathrm{a}, \mathrm{b}, *}$, T.H. Zhang ${ }^{\mathrm{a}}$, C.H. Gan ${ }^{\mathrm{a}}, \mathrm{G} . \mathrm{Yu}^{\mathrm{a}}$ \\ ${ }^{a}$ Institute of Mechanics, Chinese Academy of Sciences, 15 Beisihuanxi Road, Beijing 100080, PR China \\ ${ }^{\mathrm{b}}$ Plasma Forming Laboratory, Department of Mechanical and Materials Engineering, Florida International University, 10555 West Flagler Street, \\ EC 3232 Miami, FL 33174, USA
}

Received 29 July 2006; accepted 19 December 2006

Available online 27 December 2006

\begin{abstract}
Multi-walled carbon nanotubes (CNTs) have been successfully introduced into hydroxyapatite (HA) coatings using laser surface alloying. It is evident from transmission electron microscopy (TEM) observations that the CNTs present in the matrix still keep their multi-walled cylinder graphic structure, although they undergo the laser irradiation. Scratching test results indicated that the as-alloyed HA composite coatings exhibit improved wear resistance and lower friction coefficient with increasing the amount of CNTs in the precursor material powders. These composites have potential applications in the field of coating materials for metal implants under highload-bearing conditions.
\end{abstract}

(C) 2006 Elsevier Ltd. All rights reserved.

\section{Introduction}

Hydroxyaptite (HA) is one of the most attractive materials for human hard tissue implants because it exists as apatite in the human skeletal system and promotes the ability to bond chemically with living bone tissues [1]. However, its practical clinical applications under load-bearing conditions have been limited owing to the natural brittleness and poor strength of sintered HA. Recently, considerable research has been devoted to the development of HA acting as a coating material for titanium or other metal used in implants [2], plasma sprayed HA coating has been intensively investigated over the past two decades [2-7], in which the biocompatibility is assured by HA whilst the mechanical properties are provided by the metal substrates. Although plasma sprayed HA coatings have successful

\footnotetext{
* Corresponding author. Address: Plasma Forming Laboratory, Department of Mechanical and Materials Engineering, Florida International University, 10555 West Flagler Street, EC 3232 Miami, FL 33174, USA. Tel.: +1 305348 6533; fax: +1 3053481932 .

E-mail address: cheny@fiu.edu (Y. Chen).
}

improved on the aspects of bone attachment and integration of the implants, the long-term stability of these coatings is still a very challenging issue since these coatings trend to have uncontrollable dissolution and sometimes exhibit insufficient fracture toughness and bond strength to the metal substrate $[8,9]$, and therefore further research work needs to be carried out to improve further both the mechanical properties of HA coating and the bond strength of the HA/metal substrate interface.

Carbon nanotubes (CNTs), having unique seamless cylinders of graphite sheets either in the form of single-walled (SW) or multi-walled assemblies, have been the focus of considerable scientific research since their discovery by Iijima [10] due to their outstanding mechanical properties and excellent chemical stability. Therefore, by introducing CNTs into appropriate materials, it is postulated that the resulting composites will have largely enhanced mechanical properties compared to these unreinforced materials. Several applications have been proposed recently for CNTs, many of which adding small amount of carbon nanotubes to ceramic to produce tougher ceramic materials [11-15]. For example, Zhan et al. [11] fabricated CNT/alumina 
nanocomposite by blending dispersed single-walled carbon nanotubes with nanocrystalline alumina powders, followed by the spark plasma sintering (SPS) process. The results showed that the fracture toughness is significantly improved, three times higher that an unreinforced nanocrystalline allumina, based on indentation measurements. There are still contradictory reports on the biocompatibility of CNTs [16], although the implantation of CNTs in bone is expected to improve the mechanical properties of damaged bone tissues because CNTs are the strongest material on earth. For example, single-walled CNTs have been shown to block potassium channel activities in heterologous mammalian cell systems when applied externally to the cell surface [17], but chemically functionalized CNTs have been used successfully as substrates for neuronal growth. Therefore, evaluation of the biocompatibility and toxicity of CNTs is crucial for further research into the safety of CNTs $[18,19]$.

The microstructural characteristics, hardness and elastic modulus of the laser-surface-alloyed multi-walled CNT reinforced hydroxyapatite composite coating have been reported in our previous research work [20,21], and the results showed that the addition of CNTs has notable effect on the increase in hardness, but has no strong effect on the increase in elastic modulus, which contributes to decrease in the modulus mismatch relative to the living bone issues under the condition of guaranteeing higher strength. It is well known that the wear-resistant property is an important factor to evaluate the long-term service of these implant materials, and therefore the aim of this paper is to investigate further the distribution of these introduced CNTs and evaluate the wear resistance of HA composite coatings reinforced by CNTs using nanoscratching technique.

\section{Experimental}

Commercial hydroxyapatite powder with an average particle size ranging from 30 to $50 \mu \mathrm{m}$ and the commercially available multi-walled carbon nanotubes (MWCNTs) (Shenzhen NanoPort Company, China) with a diameter about $20 \mathrm{~nm}$ and the length from 5 to $15 \mu \mathrm{m}$ were selected as starting precursor materials. The CNTs powder was cleaned in acetone and dehydrated at $473 \mathrm{~K}$ before mixing with hydroxyapatite powder. The powder mixtures were mechanically ball-milled together in four different weight proportions, namely $0 \%, 5 \%, 10 \%$ and $20 \%$ CNTs. The substrate used for the coatings was of Ti-6Al-4V with a dimension size of $60 \times 30 \times 5 \mathrm{~mm}$. Prior to laser surface alloyed composite coatings, the substrates were preheated to $200{ }^{\circ} \mathrm{C}$, and as-alloyed coatings were cooled in the air in order to reduce the residual thermal stress. Laser surface alloying was carried out using a HL2006D Nd:YAG laser and using Ar gas as a shielding atmosphere. Laser processing parameters were selected as: laser outpower $400 \mathrm{~W}$, beam diameter $4.0 \mathrm{~mm}$ and the beam scanning speed $4 \mathrm{~mm} / \mathrm{s}$.

The cross-section of these as-alloyed CNT reinforced HA composite coatings were prepared for the metallographic samples using standard mechanical polishing procedures. Thin-foil samples for TEM observation were cut from laser surface alloyed coating, paralleling to the direction of laser beam movement. They were mechanically thinned to about $50 \mu \mathrm{m}$ and then thinned by argon ion milling. Microstructure was characterized using Neophot optical microscopy (OM), SIRION400NC field emission microscopy (FEI, Netherlands), transmission electron microscopy
(TEM) and high-resolution transmission electron microscopy (HRTEM) in a JEM-2010 operating at $200 \mathrm{kV}$ respectively. Phase constituents of the as-alloyed composite coatings were analyzed by X-ray diffraction (XRD) using a Rigaku D/max 2200 diffractometer with $\mathrm{Cu} \mathrm{K} \alpha$ radiation operated at a voltage of $40 \mathrm{kV}$, a current of $40 \mathrm{~mA}$ and a scanning rate of $5 \%$ min.

Wear tests were performed using a MTS Nano Indenter ${ }^{\circledR}$ XP system with the lateral load measurement (LFM) option. A diamond Berkovich indenter was used for face-forward scratching test, and a $700 \mu \mathrm{m}$ long scratching track was made. The normal load of indenter was linearly ramped from the minimum to the maximum of $100 \mathrm{mN}$ at scratch velocity of $10 \mu \mathrm{m} / \mathrm{s}$. A typical scratch experiment is performed in the following steps: pre-scanning with a low load of $50 \mu \mathrm{N}$ for $700 \mu \mathrm{m}$; pre-profile for $100 \mu \mathrm{m}$; Scratch profile with ramping normal load for length $500 \mu \mathrm{m}$; final profile with a normal load of $50 \mu \mathrm{N}$ for $700 \mu \mathrm{m}$. Residual morphologies of the scratch tracks of these as-alloyed HA coatings containing different amount of CNTs at the bottom stage during scratching were observed using SIRION400NC field emission scanning electron microscopy (FEI, Netherlands).

\section{Results and discussion}

X-ray diffraction (XRD) patterns of laser-surfacealloyed hydroxyapatite composite coatings containing different amount of CNTs are presented in Fig. 1. The XRD patterns of as-alloyed composite coating with 5, 10 and $20 \mathrm{wt} . \%$ CNTs in the precursor material powders (hereafter referred to as HA- $5 \%$ CNTs coating, HA-10\% CNTs coating and HA-20\% CNTs coating, respectively) show that the main constituent phases of these as-alloyed coatings are $\mathrm{HA}, \mathrm{TCP}\left(\mathrm{Ca}_{3}\left(\mathrm{PO}_{4}\right)_{2}\right), \mathrm{CaO}$ and TiC. The formation of $\mathrm{TiC}$ in the as-alloyed coatings indicates that some CNTs have reacted with elemental Ti, from Ti-6Al$4 \mathrm{~V}$, because titanium element has much larger negative heat of formation $(184.0 \mathrm{~kJ} / \mathrm{mol})$ with carbon than that of other elements in the laser-generated melt pool and the reaction of $\mathrm{Ti}$ with $\mathrm{C}$ is favored. Generally, the temperature of the laser-generated melt pool is more higher than the melting point of $\mathrm{HA}\left(1550^{\circ} \mathrm{C}\right)$, leading to the decomposition of HA to form $\alpha$-TCP $\left(\alpha-\mathrm{Ca}_{3}\left(\mathrm{PO}_{4}\right)_{2}\right)$ and TTCP $\left(\mathrm{Ca}_{4}\left(\mathrm{PO}_{4}\right)_{2} \mathrm{O}\right)$. Being an unstable phase at room temperature, $\alpha$-TCP naturally transform to $\beta$-TCP $\left(\beta-\mathrm{Ca}_{3}\left(\mathrm{PO}_{4}\right)_{2}\right.$,

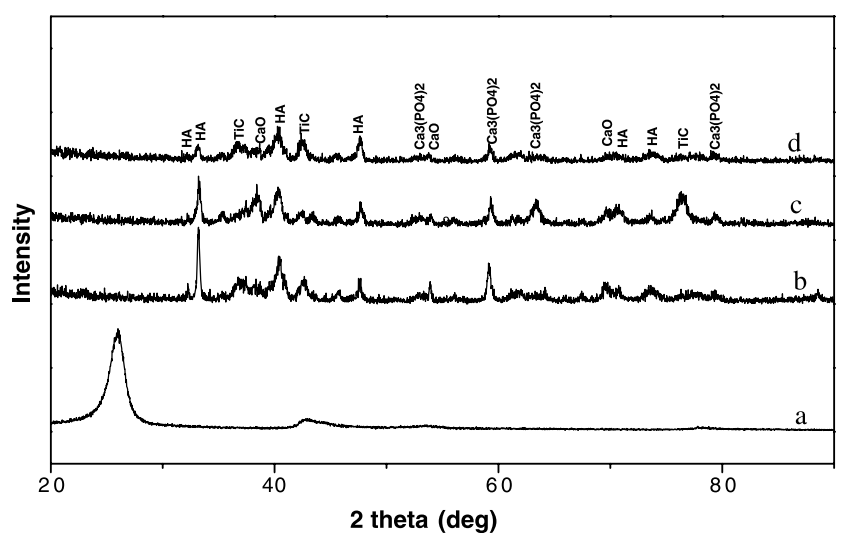

Fig. 1. XRD patterns of (a) pure carbon nanotubes, HA composite coatings containing different amount carbon nanotubes (b) HA-5\% CNTs coating, (c) HA-10\% CNTs coating and (d) HA-20\% CNTs coating. 
a stable phase at room temperature) at about $1100{ }^{\circ} \mathrm{C}$ [22]. Concerning the TTCP phase, it would further decompose to form $\mathrm{HA}$ and $\mathrm{CaO}$ [23].

The morphologies of the CNTs distributed in the hydroxyapatite matrix are indicated in Fig. 2. Fig. 2a shows the CNTs are slightly bend, and higher magnification picture shown in Fig. $2 \mathrm{~b}$ indicates that CNTs possess bamboo-knot-like defects, implying the CNTs are damaged when they undergo high temperature produced by laser irradiation. It is interesting that the CNT bundles consisted of some straight, parallelly aligned CNTs that appear to be sintered together, as shown in Fig. 2c. Meanwhile, some CNTs are also found to be saw-toothed-like, as shown in Fig. 2d. The formation of saw-toothed-like CNT is attrib- uted to the in situ reaction of Ti element on the CNT surface under high-temperature conditions. These saw-tooth CNTs anchor into HA matrix and lead to a significantly higher stress for the pullout and debonding of CNT, which can improve further the composite's fracture toughness. It is clearly seen from Fig. 2e that some CNT bundles are welded on the matrix tip, which is very similar to the experimental results reported in the reference [24]. Additionally, large amount of CNTs are entangled each other, as indicated in Fig. 2f. Although some CNTs are damaged more or less under high temperature produced by laser irradiation, it can be confirmed that the CNTs still keep their multi-walled cylinder graphic structure intact due to both the high thermal stability and high chemical stability
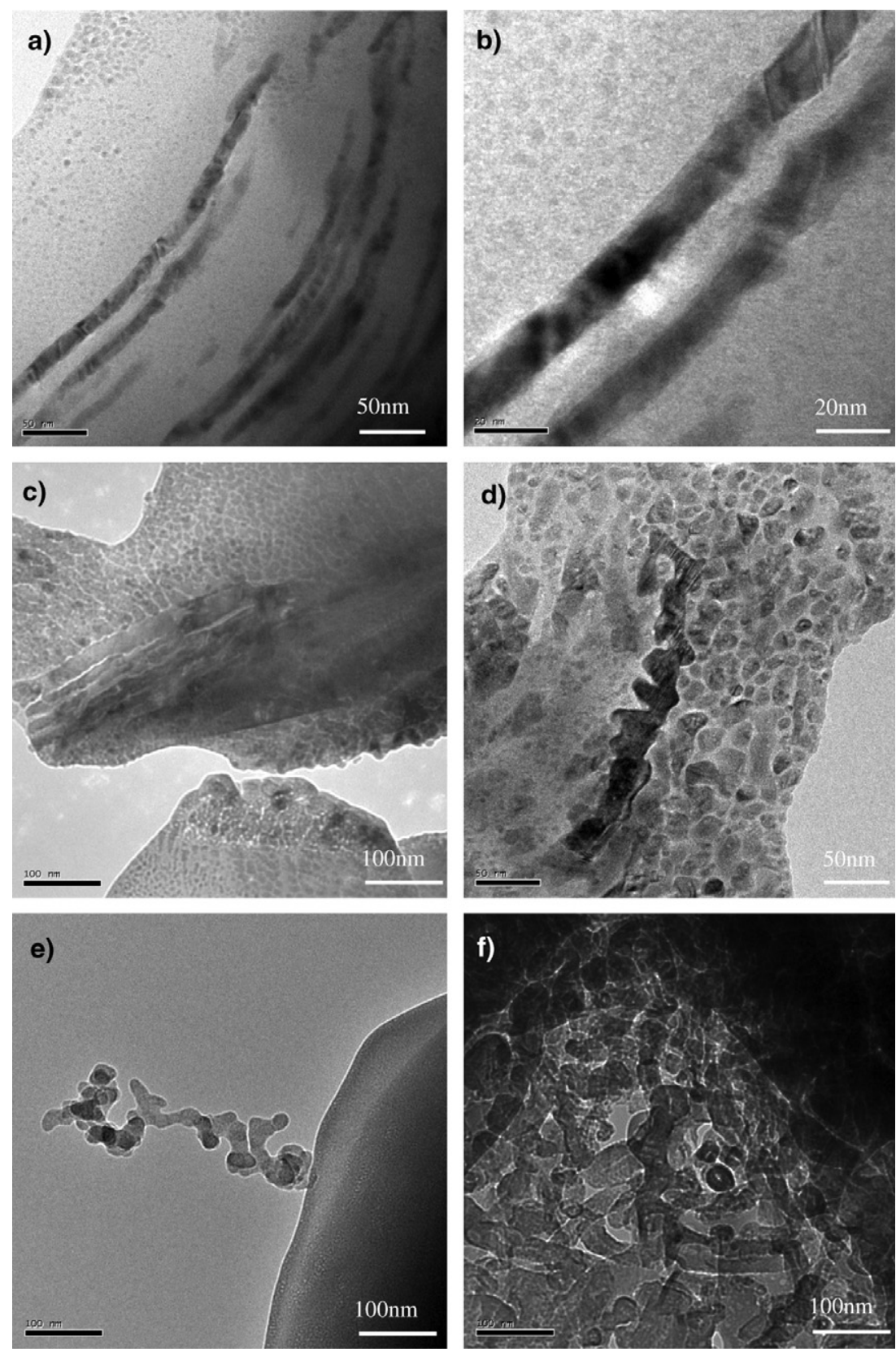

Fig. 2. TEM images showing distribution morphologies of introduced CNTs. 
of the CNTs $[25,26]$. It proves that CNTs have been successfully introduced into HA matrix and the addition CNTs might still possess their excellent mechanical properties.

The depth variation of as-alloyed HA composite coating containing different amount of CNTs is shown in Fig. 3, which is as a function of displacement during nanoscratching (scratch depth) and post-depth (residual depth) (hereafter referred to as "D-D curves"). In general, the cracking or delaminating of a coating and/or film is signaled by a sudden increase in either friction coefficient and scratch-depth curves [27-29]. The D-D curves of asalloyed HA coating, as shown in Fig. 3a, indicates the scratch process could be divided into three stages, i.e., fully elastic recovery (marked as A in Fig. 3a), plastic deformation (marked as B in Fig. 3a), and delaminating and pulling-off of coating material (marked as $\mathrm{C}$ in Fig. 3a). Compared with the D-D curves of as-alloyed HA coatings, D-D curves of these as-alloyed CNT reinforced HA composite coatings all have fully elastic recovery stage, as shown in Fig. 3b-d. However, for these as-alloyed CNT/ HA composite coatings, the scratch-depth curves and friction coefficient curves fluctuate strongly during the scratch test so as to be difficult to distinguish the plastic deformation stage and delaminating stage, as shown in Figs. 3b-d and $4 \mathrm{~b}-\mathrm{d}$. Authors think that it might be result from the inhomogeneous microstructure of the HA composite coatings. As is known, the agglomeration of as-prepared CNTs in the precursor materials powders occurs due to their high surface energy, and therefore the introduced CNTs often agglomerate in the HA matrix (Fig. 2f). Inhomogeneous distribution of the introduced CNTs would lead to the inhomogeneous distribution of the in situ formed $\mathrm{TiC}$ phase. In the process of scratch test, resistance force upon the diamond tip would be different due to the nonuniform distribution of introduced CNTs and in situ formed TiC phase in the HA matrix, making the scratch-depth curves and friction coefficient curves fluctuate. Also, no delaminating or pulling-off of coating materials is observed at the start section of the scratch test for all as-alloyed coating containing different amount CNTs. As the amount of CNTs increases in the precursor material powders, the friction coefficient of as-alloyed CNT reinforced HA composite coating decreases, as shown in Fig. 4. It is suggested that lubrication effect of these as-alloyed composite coating is improved.
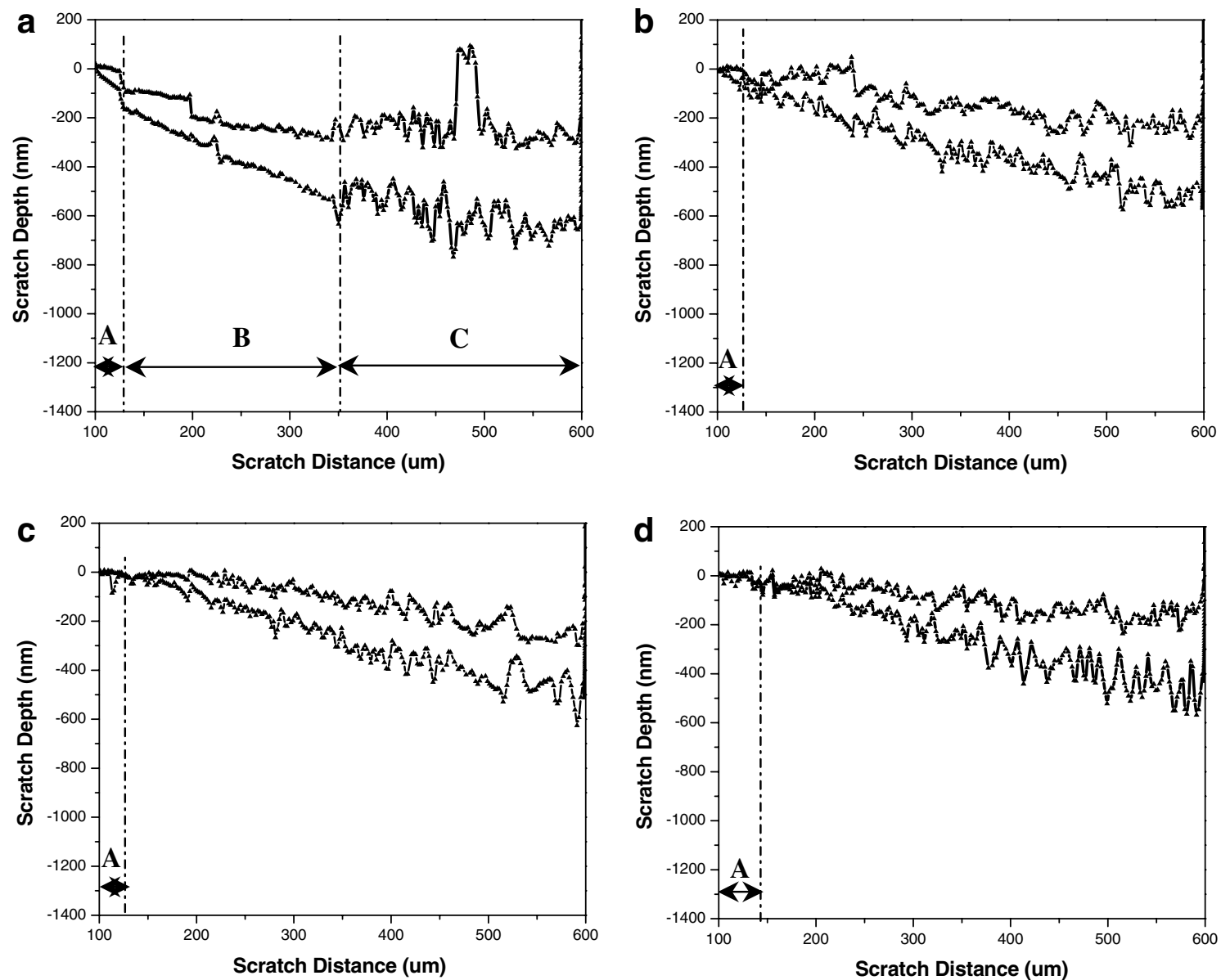

Fig. 3. D-D curves of as-alloyed HA coatings containing different amount of CNTs (a) CNT-free coating, (b) HA-5\% CNTs coating, (c) HA- $10 \%$ CNTs coating and (d) HA-20\% CNTs coating. 

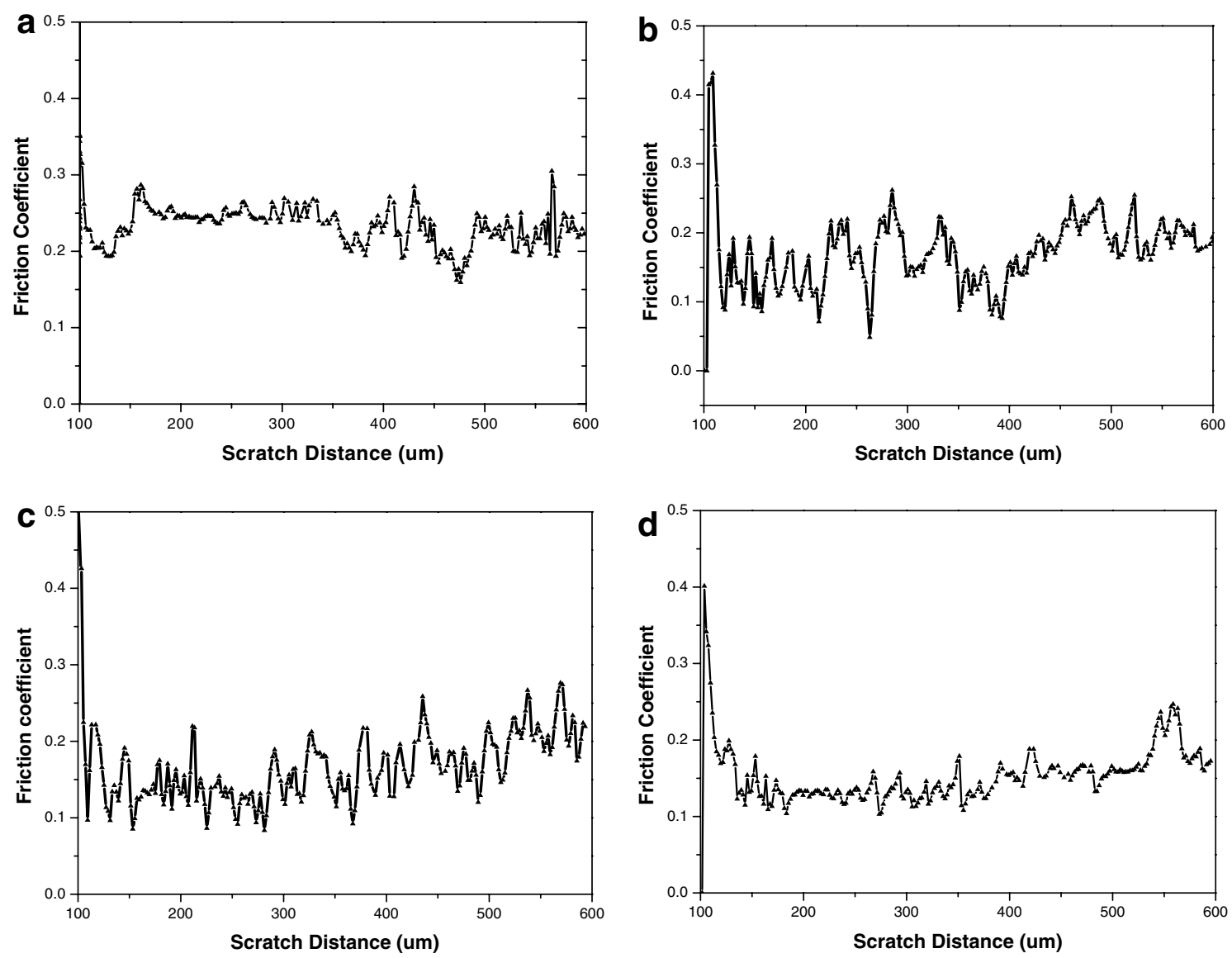

Fig. 4. Friction coefficient variation during scratching of as-alloyed HA coatings containing different amount of CNTs (a) CNT-free coating, (b) HA-5\% CNTs coating, (c) HA-10\% CNTs coating and (d) HA-20\% CNTs coating.

Field emission SEM images of the morphologies of scratch tracks of these as-alloyed HA coating containing different amount of CNTs are presented in Fig. 5. As shown in Fig. 5a, the morphology of the scratch track for the as-alloyed HA coating is deep ploughing groove with many fine cracks emanating from the trace of diamond tip, indicating that the diamond tip can easily penetrate into the surface of HA coating and subsequently micro-cut HA coating during scratch test. As is known, the face of Berkovich indenter is towards the scratching direction in the scratching experiments, leading to the stress concentration induced at two edges of the tip, and therefore the stress concentration increases gradually in the process of the ramping load scratch. After stress exceeds the fracture toughness of the as-alloyed coating, cracks form along the edges of the indenter tip to release the stress. Subsequently, the crack density and/or crack length increase further to make these cracks coverage and lead to the secondary cracks formation, which usually parallel to the scratching direction and would induce the delaminating of coating materials.

When the amount of CNTs in precursor material powders increases to $5 \mathrm{wt} . \%$, the scratch-track morphology is shallower than that of HA coating, and the crack density clearly goes down, as shown in Fig. 5b. As the amount of CNTs in the precursor material powder increases further, the scratch-track morphologies of the as-alloyed HA composite coatings become more shallow, and the crack density and crack length all decrease, as shown in Fig. 5c and $d$ respectively. This phenomenon strongly suggests that the as-alloyed HA composite coatings become more difficult to be ploughed and plastically deformed during scratch test, which is attributed to the combination strengthening effect of addition CNTs and in situ formed TiC phase. Compared with the scratch-track morphologies of the asalloyed HA composite coatings containing different amount of CNTs, little fine crack is found along the trace of the indenter tip at the bottom of scratch test for the CNTs reinforced HA composite coating with a precursor material powder of HA-20\% CNTs (wt.\%). The results strongly implies the addition of CNTs into HA matrix lead to increase in the strength of the HA composite coatings to resist the stress concentration induced at the edges of the tip. Meanwhile, the addition of CNTs also results in the increase in hardness of the CNT reinforced HA composite coatings [20], making it difficult to be penetrated by the diamond tips. It is well known that the CNTs are extraordinarily flexible under large strains and resist failure under repeated bending [30], and therefore the addition of CNTs in the HA matrix allows the fracture energy absorption or 

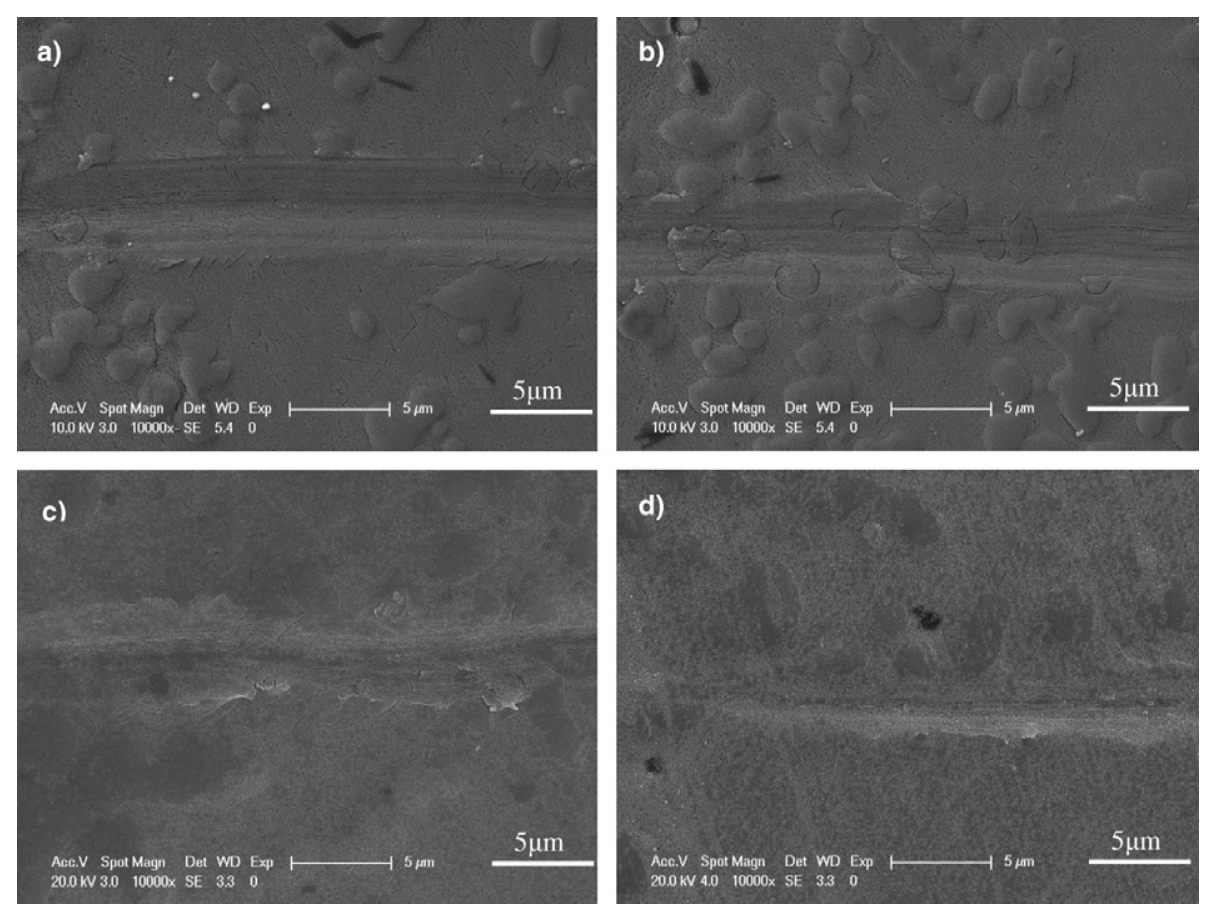

Fig. 5. Field emission SEM images of morphologies of the scratch tracks (scratch performed from left to right) of as-alloyed HA coatings containing different amount of CNTs at the bottom stage during scratching (a) CNT-free coating, (b) HA-5\% CNTs coating, (c) HA-10\% CNTs coating and (d) HA$20 \%$ CNTs coating.

dissipation under stress and significantly improve the fracture toughness of as-alloyed HA composite coatings. Further work regarding as the fracture toughness and fracture mechanisms of the CNT reinforced HA composite coating by using other technique is in progress.

\section{Conclusions}

Multi-walled carbon nanotubes (CNTs) have been successfully introduced into HA coatings using laser surface alloying. TEM observation illustrated that the introduced CNTs keep their original cylinder graphic structure even if they undergo high-temperature damage. The addition of CNTs can improve the wear resistance of HA composite coatings, which might be attributed to the increase in the hardness, strength and fracture toughness. We believe that these composites might have potential applications in the field of coating materials for metal implants under highload-bearing conditions.

\section{Acknowledgements}

The authors acknowledge the financial support of the National Natural Science Foundation of China (Grant Nos. 59971003, 10572142 and 10432050). Meanwhile, the authors wish to thank Prof. Arvind Agarwal at the Plasma forming Laboratory, Florida International University, for his polishing this manuscript.

\section{References}

[1] Tadic D, Peters F, Epple M. Continuous synthesis of amorphous carbonated apatites. Biomaterials 2002;23(12):2553-9.

[2] Lynn AK, DuQuesnay DL. Hydroxyapatite-coated Ti-6Al-4V Part 1: the effect of coating thickness on mechanical fatigue behavior. Biomaterials 2002;23(9):1937-46.

[3] Cheang P, Khor KA, Teoh LL, Tam SC. Pulsed laser treatment of plasma-sprayed hydoxyapatite coatings. Biomaterials 1996;17(19): 1901-4.

[4] Ducheyne P, Beight J, Cuckler J, Evans B, Radin S. Effect of calcium phosphate coating characteristics on early post-operative bone tissue ingrowth. Biomaterials 1990;11(8):531-40.

[5] Weng J, Liu X, Zhang X, Ma Z, Ji X, Zyman Z. Further-studies on the plasma-sprayed amorphous phase in hydroxyapatite coatings and its deamorphization. Biomaterials 1993;14(8):578-82.

[6] Dalton JE, Cook SD, Thomas KA, Kay JF. The effect of operative fit and hydroxyapatite coating on the mechanical and biological response to porous implants. J Bone Joint Sug 1995;77A(1):97110.

[7] Klein CPAT, Patka P, Van der Lubbe HBM, Wolke JGC, De Grook K. Plasma sprayed coating of tetracalium phosphate, hydoxyapatite, and $\alpha$-TCP on titanium alloy: an interface study. J Biomed Mater Res 1991;25(4):53-65.

[8] Kay JF. Calcium phosphate coatings for dental implants. Dent Clin North Am 1992;36:1-18.

[9] Zeng HT, Lacefield WF. XPS, EDX and FTIR analysis of pulsed laser deposited calcium phosphate bioceramic coatings: the effects of various process parameters. Biomaterials 2000;21(1):23-30.

[10] Iijima S. Helical microtubules of graphitic carbon. Nature 1991; 354(6384):56-8.

[11] Zhang GD, Kuntz JD, Wan JL, Mukherjee AK. Single-wall carbon nanotubes as attractive toughening agents in alumina-based nanocomposites. Nat Mater 2003;2(1):38-42.

[12] Baughman RH, Zakhidov AA, de Heer WA. Carbon nanotubes - the route towards applications. Science 2002;297(5582):787-92. 
[13] Thostenson ET, Ren ZF, Chou TW. Advances in the science and technology of carbon nanotubes and their composites: a review. Compos Sci Technol 2001;61(13):1899-912.

[14] Dai HJ. Carbon nanotubes: Opportunities and challenges. Surf Sci 2002;500(1-3):218-43.

[15] Lupo F, Kamalakaran R, Scheu C, Grobert N, Ruhle M. Microstructural investigations on zirconium oxide-carbon nanotube composites synthesized by hydrothermal crystallization. Carbon 2004;42(10):1995-9.

[16] Zanello LP, Zhao B, Hu H, Haddon RC. Bone cell proliferation on carbon nanotubes. Nano Letter 2006;6:562-7.

[17] Park KH, Chhowalla M, Iqbal Z, Sesti F. Single-walled carbon nanotubes are a new class of ion channel blockers. J Biol Chem 2003;278:50212-6.

[18] Hu H, Ni YC, Montana V, Haddon RC, Parpura V. Chemically functionalized carbon nanotubes as substrate for neuronal growth. Nano Letter 2004;4(3):507-11.

[19] Balani K, Anderson R, Laha T, Andara M, Tercero J, Crumpler E, et al. Plasma-sprayed carbon nanotube reinforced hydroxyapatite coatings and their interaction with human osteoblasts in vitro. Biomaterials 2007;28(4):618-24.

[20] Chen Y, Gan CH, Zhang TN, Yu G, Bai PC, Kaplan A. Lasersurface-alloyed carbon nanotubes reinforced hydroxyapatite composite coating. Appl Phys Letter 2005;85:251905(1-3).

[21] Chen Y, Zhang YQ, Zhang TH, Gan CH, Zheng CY, Yu G. Carbon nanotube reinforced hydroxyapatite composite coatings produced by laser surface alloying. Carbon 2006;44(1):37-45.
[22] Li HX, Marquis PM. Effect of teat treatment on the microstructure of plasma-sprayed hydroxyapatite coating. Biomaterials 1993;14(8): 64-68.

[23] Yang CY, Wang BC, Chang E, Wu JD. The influence of plasma spraying parameters on the characteristics of hydroxyapatite coating: a quantitative study. J Mater Sci 1995;6(5):249-57.

[24] Zhang Y, Ichihashi T, Landree E, Nihey F, Iijima S. Heterostructure of single-walled carbon nanotubes and carbide nanorods. Science 1999;285(9):1719-22.

[25] Ebbesen TW, Ajayan PM. Laser-scale synthesis of carbon nanotubes. Nature 1992;358(6383):220-2.

[26] Iijima S, Ichihashi T. Single-shell carbon naotubes of 1-nm diameter. Nature 1993;363(6430):603-5.

[27] Bhushan B, Gupta BK, Azarian A. Nanoindentation, microscratch, friction and wear studies of coatings for contact recording applications. Wear 1995;181(2):743-58.

[28] Gupta BK, Bhushan B. Mechanical and tribological properties of hard carbon coatings for magnetic recording heads. Wear 1995; 190(1):110-22.

[29] Huang LY, Xu KW, Lu J, Guelorget B. Nano-scratching process and fracture mechanisms of amorphous carbon films. Wear 2003;254(10): $1032-6$.

[30] Falvo MR, Clary GJ, Taylor RM, Chi V, Brooks Jr FP, Washburn S. Bending and buckling of carbon nanotubes under large strain. Nature 1997;389(6651):582-4. 